\title{
Gestión de la historia clínica electrónica y la mejora de la calidad de atención al paciente en el servicio de Odontología del hospital Hermilio Valdizán
}

\author{
Management of the electronic clinical history and improvement of the quality of patient \\ care in the dentistry department of the Hermilio Valdizan hospital
}

\author{
Úrsula Margarita Gutiérrez-Samaniego (ib ${ }^{1 a}$
}

\section{RESUMEN}

Objetivo: Verificar la relación de dependencia lineal directa de la implementación de la Historia Clínica Electrónica (HCE) en la Mejora de la Calidad de Atención al Paciente en el Servicio de Odontología en el Hospital Hermilio Valdizán (HHV). Diseño: Tipo aplicada o práctica. Análisis estadístico: Coeficiente correlación de Spearman. Materiales y métodos: Aplicación de cuestionario a 14 personales del servicio de odontología y encuestas a 70 pacientes continuadores durante el periodo de diciembre 2020 y enero 2021. Resultados: existe evidencia estadística suficiente a un nivel alto significativo del $85.5 \%$, que la implementación de la Historia Clínica Electrónica (HCE) se correlaciona linealmente con la mejora de la Calidad de la Atención en el Servicio de Odontología. Conclusiones: Priorizar la implementación de la HCE como Plan Piloto y en función a resultados a una mayor escala implementar a nivel de todos los servicios del HHV.

Palabras clave: Historia Clínica Electrónica; Gestión de Servicios de Salud y Mejora de Calidad de Servicio. (Fuente: DeCS BIREME)

\section{ABSTRACT}

Objective: To verify the direct linear dependence relationship of the implementation of the Electronic Health Record (EHR) in the Improvement of the Quality of Patient Care in the Dentistry Service at the Hermilio Valdizan Hospital (HHV). Design: Applied or practical type. Statistical analysis: Sperman's correlation coefficient. Materials and methods: Questionnaire application to 14 dental service personnel and surveys to 70 continuing patients during the period of December 2020 and January 2021. Results: there is sufficient statistical evidence at a significant high level of $85.5 \%$, that the implementation of the Electronic Health Record (EHR) is linearly correlated with the improvement of the Quality of Care in the Dentistry Service. Conclusions: Prioritize the implementation of the HCE as a Pilot Plan and based on results on a larger scale, implement at the level of all the HHV services.

Keywords: Electronic Medical Record; Health Services Management and Service Quality Improvement. (Source: MeSH NLM)

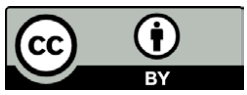

Citar como: Gutiérrez Samaniego, Úrsula. Gestión de la historia clínica electrónica y la mejora de la calidad de atención al paciente en el servicio de Odontología del hospital Hermilio Valdizán. KIRU. 2021; 18(2):82-88. https://doi.org/10.24265/kiru.2021.v18n2.02 


\section{INTRODUCCIÓN}

El objetivo de la presente investigación es verificar la relación de dependencia lineal directa de la implementación de la Historia Clínica Electrónica en la mejora de la Calidad de la Atención al Paciente en el Servicio de Odontología en el Hospital Hermilio Valdizán (HHV).

Las atenciones por consulta externa en el Hospital Hermilio Valdizán presentan dificultades en el día a día, siendo una de ellas la no disponibilidad oportuna de la historia clínica manuscrita durante la atención de pacientes; lo que genera una duplicidad de información, alteración del orden, falta de continuidad en la atención y sobre todo demora o reprogramación de la consulta por falta de la Historia Clínica que genera insatisfacción a los pacientes ${ }^{(1)}$.

Según el informe de los resultados de la Encuesta del usuario externo, metodología Servqual (2), realizado por el HHV en el 2019, de un total de 318 encuestados, concluyen un alto grado de insatisfacción del orden del $59 \%$ en relación al servicio en consulta externa y $65 \%$ en relación a la no disposición de la Historia Clínica en el Consultorio para su atención (Ver figura 1). Así como también, en el Plan Cero Colas 2020 del HHV ${ }^{(3)}$, reflejan la situación problemática donde refieren que existen demoras en la disponibilidad de las historias clínicas en consulta externa.

En el Servicio de Odontología del HHV presenta esta problemática, por lo que se plantea la necesidad de implementación de un sistema digital de información: Historia Clínica Electrónica (HCE).

La Historia Clínica Electrónica (HCE) se define como "...aquella que reside en un sistema electrónico específicamente diseñado para recolectar, almacenar, manipular y dar soporte a

Tabla 1. Grado de satisfacción e insatisfacción del usuario en consulta externa dimensión fiabilidad

\begin{tabular}{|c|c|c|c|c|}
\hline \multirow[t]{2}{*}{ Preguntas } & \multicolumn{2}{|c|}{ Satisfecho } & \multicolumn{2}{|c|}{ Insatisfecho } \\
\hline & $\mathrm{n}$ & $\%$ & $\mathrm{n}$ & $\%$ \\
\hline $\begin{array}{l}\text { P1:Que el personal de informes le oriente y explique de } \\
\text { manera clara sobre los pasos o tramites para la atención } \\
\text { en consulta externa }\end{array}$ & 112 & 35.3 & 205 & 64.7 \\
\hline $\begin{array}{l}\text { P2: Que la consulta con el medico se realice en el horario } \\
\text { respetando la programación y el orden de llegada }\end{array}$ & 124 & 39 & 194 & 61 \\
\hline $\begin{array}{l}\text { P3: Que la atención se realice respetando la } \\
\text { programación }\end{array}$ & 122 & 38.4 & 196 & 61.6 \\
\hline \multicolumn{5}{|l|}{ y el orden de llegada } \\
\hline $\begin{array}{l}\text { P4: Que su historia clínica se encuentre disponible en el } \\
\text { consultorio para su atención }\end{array}$ & 111 & 34.9 & 207 & 65.1 \\
\hline \multirow{2}{*}{$\begin{array}{l}\text { P5: Que las citas estén disponibles y se obtengan } \\
\text { con facilidad }\end{array}$} & 110 & 34.6 & 208 & 65.4 \\
\hline & 579 & 36.4 & 1010 & 63.6 \\
\hline
\end{tabular}


los usuarios en cuanto a proveer accesibilidad a datos seguros y completos, alertas, recordatorios y sistemas clínicos de soporte para la toma de decisiones, brindando información clínica importante para el cuidado de los pacientes..." (Instituto de Medicina, Dick y Stein, 1991) ${ }^{(4)}$.

Ampliando esta definición, Dick, Stein y Detmer ${ }^{(4)}$, mencionan:

1. Colección longitudinal de información electrónica sobre la salud de las personas donde la información sobre salud es definida como información pertinente a la salud de un individuo, o la información de los cuidados de salud provistos a un individuo, por medio de cualquier miembro del equipo de salud.

2. Tiene la posibilidad de dar acceso electrónico inmediato a la información de salud personal o población sólo a los usuarios autorizados.

3. Provee las bases de conocimiento y sistemas de soporte para la toma de decisiones que mejoren la calidad, seguridad y eficiencia de la atención de los pacientes.

4. Tiene como objetivo primordial de dar soporte para la eficiencia de los procesos de cuidados de salud. Representa un conjunto de sistemas que deben estar altamente integrados y que requieren una significativa inversión de tiempo, dinero, cambio de procesos y reingeniería del factor humano.

Esta herramienta digital trae consigo una serie de ventajas a los operadores de los sistemas de salud y pacientes generando impactos positivos en los procesos de atención en consulta externa. Toscano ${ }^{(5)}$ y Donato (6) mencionan que las Tecnologías de la Información (TIC) abren nuevas oportunidades para la moderización institucional, proveen de herramientas que ayudan a mejorar la disponibilidad, la calidad y la seguridad en la atención de la salud. Así como también Cóndor ${ }^{(7)}$ señala que la HCE tiene gran potencial en mejorar los servicios de salud y es necesario desarrollarla según necesidades, implementarla y evaluarla. El uso de HCE está cambiando cómo los profesionales de salud y pacientes generan y procesan información.

Un estudio realizado por Tuanama ${ }^{(8)}$, hace referencia a la transición del registro médico en papel a su formato digital que representa en los centros de salud en los operadores del sistema, y según la experiencia de su implementación, existe un reconocimiento generalizado por parte del personal de salud de la utilidad y la necesidad de migrar a herramientas tecnológicas para generar la data clínica de los pacientes.

\section{MÉTODOS:}

En el periodo de diciembre 2020 y enero 2021 se realizó un estudio de investigación del tipo aplicada o práctica, donde se implementó la HCE en un servicio específico del HHV, basado en una necesidad existente. Los criterios de selección del presente estudio fueron el personal del Servicio de Odontología, tanto asistencial como administrativo en calidad de proveedores del servicio, siendo un total de 14 personas y por otro lado los pacientes y/o familiares en calidad de receptores del servicio con un total de 70 pacientes continuadores, los cuales firmaron un consentimiento informado aprobado por el Comité de Ética y fueron debidamente categorizados según el Manual de Codificación de Diagnósticos en la Atención de Pacientes (9), correspondiendo a pacientes esquizofrénicos, trastornos de ansiedad, episodio depresivo, trastornos de alcoholismo y trastornos hipercinéticos entre los principales.

Se utilizaron tres instrumentos para la recolección de datos: 1. Cuestionario aplicado al personal de salud del servicio de odontología, 2. Encuestas a los pacientes de consulta externa del servicio de odontología y 3. Análisis documentario. El análisis estadístico utilizado fue la prueba de coeficiente de correlación de Spearman para evaluar la hipótesis general y específicas, considerando que las variables son de escala ordinal.

\section{RESULTADOS:}

Los resultados sobre el prototipo de HCE, el $71 \%$ del personal asistencial y administrativo considera crítico la necesidad de disponer de la historia clínica para una atención oportuna, siendo éste el principal problema actual en el HHV.

Otro aspecto de vital importancia en las historias clínicas es la necesidad que contenga información completa integra y actualizada, para poder realizar 
un buen diagnostico, tartamiento o referencia médica en función de la información consignada, así como también, toda la información del paciente debe poder guardarse en un banco de datos según lo establece la norma técnica, éstas situaciones han sido reconocidas por el $78 \%$ de personas entrevistadas.

En cuanto a la interoperabilidad, es considerado un aspecto clave de las HCE por el $78.6 \%$ de los entrevistados, porque permite que ésta pueda ser compartida con otras bases de datos para disponer de información dentro de un sólo sistema y así facilitar su atención en cualquier punto de la red del sistema de salud. Por otra parte, un $71 \%$ de los entrevistados considera que las HCE constituyen un soporte importante para una eficiente gestión clínica y en definitiva está relacionada con otros factores como la infraestructura, equipamiento y capacitación.

Con respecto a los resultados del cuestionario sobre la implementación de la HCE y su relación con la calidad de atención, el $80 \%$ de entrevistados considera que es un factor crítico en los sistemas de atención actual el tiempo de búsqueda de las historias clínicas, a su vez el $100 \%$ de encuestados considera clave realizar validaciones previas de acceso a las HCE, tomando en cuenta que la información consignada allí es de carácter personal y su uso debe ser restringido solo a personas debidamente autorizadas por el mismo paciente. Reafirmando con un $98 \%$ de los pacientes encuestados que aseguran que la integridad del sistema es un factor importante para una atención segura a ellos.

Con el uso de la HCE, el $100 \%$ de los pacientes considera que se permite respetar la hora de programación de su cita, solucionando uno de los principales problemas actuales con las historias clínicas manuscritas al estar siempre disponibles en línea a través de una plataforma informática, así como también el $97 \%$ consideran que la HCE agilizará los resultados de otros servicios como Laboratorio, Radiología, Patología y otros centralizando toda la información del paciente en una misma plataforma.

El $100 \%$ de los pacientes entrevistados considera que la implementación de las HCE se sentirá más satisfecho con el servicio brindado al mayor acceso, oportunidad, disponibilidad e información completa a través de esta plataforma informática. Tanto el personal de odontología y los pacientes del servicio, consideran en un $100 \%$ que la plataforma informática de las HCE deber ser rápido, amigable y de fácil manejo, conllevando a mejorar la calidad de los servicios de atención de salud. Así como también coinciden que la HCE debe poder ser visualizada desde cualquier punto de la red como un medio de facilitar su atención médica.

La comprobación de la hipótesis general del presente estudio, con la prueba coeficiente de correlación Rho de Spearman, para conocer el grado de asociación entre las variables, fue que un nivel de significancia de 0.05 se tiene evidencia estadística para rechazar $\mathrm{Ho}$, por lo tanto, se concluye que a un nivel significativo alto del $85.8 \%$, la implementación de la historia clínica electrónica se correlaciona directamente con la mejora de la calidad de atención del servicio de odontología del Hospital Hermilio Valdizán. (Tabla 2).

\section{DISCUSIÓN:}

La implementación de la HCE en el Perú está regida por el marco legal establecido por la Ley Nro. $30024^{(10)}$; Ley que crea el Registro Nacional de Historia Clínica Electrónica y su reglamento ${ }^{(11)}$, por la Norma Técnica Nro. 139MINSA/2018/DGAIN Norma Técnica de Salud para la Gestión de la historia clínica del Ministerio de Salud (12) y por Norma Técnica Nro. 022MINSA/DGSP-V.01 Norma Técnica de la Historia (13) 
Tabla 2: Rho Spearman Hipótesis General HCE

\begin{tabular}{|l|r|}
\hline $\mathrm{N}$ & $\mathbf{7 0}$ \\
\hline Sum D2 & 8115.50 \\
\hline Coeficiente Spearman & $\mathbf{0 . 8 5 8 0 0 8 9 2 3}$ \\
\hline
\end{tabular}

\begin{tabular}{|l|r|}
\hline Valor Critico & 70 \\
\hline $\mathrm{N}$ & 68 \\
\hline $\mathrm{N}-2$ & 0.05 \\
\hline Alpha (5\%) & 1.995468931 \\
\hline Valor Critico & 7.036919301 \\
\hline Estadistico Prueba T & \\
\hline
\end{tabular}

Dentro del Plan Estratégico Institucional-PEI MINSA 2019-2023 ${ }^{(14)}$, establece en su objetivo institucional 6: "Mejorar la toma de decisiones, la prestación de servicios públicos, el empoderamiento y la satisfacción de la población a través del gobierno digital en salud", acorde al uso de una plataforma informática como la HCE, los beneficios se reflejan en la mejora en la accesibilidad, opotunidad y calidad de los servicios de salud para los usuarios, en la disponibilidad de recursos y simplificación administrativa que enfrentan los profesionales y personal técnico y podrá dedicar mayor tiempo al campo asistencial.

Así mismo en el Plan Operativo Institucional POI $2020 \mathrm{HHV}^{(15)}$, determina en sus líneas de acción AEI 06.02 "Información de la Historia Clínica Electrónica de usuarios en el sistema de salud disponible", priorizando la implementación de las HCE en el HHV, como parte de su estrategia institucional y logro de objetivos institucionales.

Los resultados de la investigación coinciden con los de Cóndor ${ }^{(7)}$, llegando a la conclusión que la implementación de la HCE en una institución de salud, logra la satisfacción del usuario final, brindando utilidad en la atención de sus pacientes, como es, en la reducción de tiempo en el llenado de datos, y evitar la omisión de estos ultimos.
El tiempo de atención es considerado un factor crítico por los usuarios del servicio relacionado con la desorganización de las historias clínicas que genera demoras en la atención, coincidiendo con Véliz ${ }^{(16)}$, que los resultados obtenidos con un sistema informático reducen costos en relación al material, mejora el tiempo de atención y presenta calidad de atención.

Similar resultado sobre el manejo y búsqueda de las historias clínicas, considerado principal problema en el HHV de no disponerla durante su atención y coincidiendo con Toscano ${ }^{(5)}$ que realizó un estudio sobre el análisis de la importancia de la información que se maneja en la salud para poder cumplir con las premisas de un servicio de calidad, de eficiencia por parte de los prestadores de servicios y establecer la viabilidad de un sistema de Historias Clínicas Electrónicas en el sistema de salud pública, mostrando resultados favorables con la implementación de la HCE mejorando la productividad y eficiencia desde su aplicación. Así como también Poceiro ${ }^{(17)}$, señala la importancia de los servicios de salud tanto públicos y privados y el impacto que las tecnologías de la información han tenido sobre ellos, simplificando procesos, gestión segura de información sensible y mejora de servicios de atención sanitari 
Uldrich ${ }^{(18)}$, señala los "tres impactos de la salud digital en las atenciones de la salud", que las nuevas tendencias en el cuidado de la salud conllevan a abordar desafíos claves que impactan en la vida de los pacientes.

Sobre la base de las experiencias de implementación de HCE en nuestro país, los impactos que podrían generarse son: mejor manejo clínico del paciente, empoderamiento del paciente y mejora del acceso de pacientes a los servicios de salud.

Dentro de las limitaciones de este estudio, se encuentra que, por el diagnóstico de salud mental de los pacientes del Servicio de Odontología, en algunos casos se tuvo que contar con el apoyo de los apoderados y/o familiares para la realización de los cuestionarios, así mismo también la situación actual de la emergencia sanitaria por el covid 19 ha conllevado a sostener algunas limitaciones para captar a mayor cantidad de pacientes. Y por último se han visto limitadas las coordinaciones con otras instituciones de salud que cuentan con este servicio de historias clínicas electrónicas por el tema de la emergencia sanitaria.

\section{CONCLUSIONES:}

1. La implementación de la historia clínica electrónica se correlaciona directamente con la mejora de la calidad de atención del servicio de odontología del Hospital Hermilio Valdizán.

2. La HCE es considerado uno de los principales avances tecnológicos de los últimos años en atención asistencial de salud. Facilitando tanto el trabajo de los profesionales de la salud, técnicos y personal administrativo.

3. El MINSA cuenta con una Agenda Digital del Sector Salud 2020-2025, priorizando la transformación digital a nivel nacional a través de la HCE, telesalud y otras enfocadas a mejorar el acceso y atencion con calidad de los servicios de salud.

4. La gestión de la HCE se encuentra regulada por el MINSA, la misma que viene implementando progresivamente a todos los centros de salud públicos para un manejo estandarizado de la plataforma tecnológica.

5. El HHV ha priorizado dentro de sus planes estratégicos y operativos la implementación de la HCE, como una forma de mejorar los niveles de atención de pacientes en consulta externa para alcanzar el bienestar individual y colectivo.

6. La importancia del la HCE en el Servicio de Odontología en el HHV radica en la incorporación de un sistema digital de disponibilidad y accesibilidad inmediata, que contiene toda la información médica relevante y actualizada que hace posible un adecuado diagnóstico, tratamiento médico del paciente y reduce la posibilidad errores.

7. La implementación de la HCE a nivel de un Plan Piloto en el Servicio de Odontología del $\mathrm{HHV}$, se correlaciona con la implementación progresiva en los demás Servicios del HHV de acuerdo a los estándares del RENHICE, que contribuya con la mejora de la calidad de atención en el Servicio de Odontología y los otros Servicios Médicos del HHV, logrando la satisfacción de los pacientes.

\section{Fuente de financiamiento: autofinanciado}

Conflictos de interés: no hubo ningún conflicto de interés.

\section{REFERENCIAS BIBLIOGRÁFICAS:}

1. Gutiérrez U. Implementación de Historia Clínica Electrónica en la Mejora de la Calidad de Atención del Servicio de Odontología del Hospital Hermilio Valdizán Lima - Perú. [Maestría]. Universidad Nacional Mayor de San Marcos; 2021.

2. Informe de los Resultados de la Encuesta del usuario Externo, Metodología Servqual 2019. Lima: Hospital Hermilio Valdizán; 2019.

3. Plan Cero Colas 2020 del HHV. Resolución Directoral Nro. 66-DG/HHV/2020, Lima: Hospital Hermilio Valdizán; 2020.

4. Carnicero J, Fernández A. Manual de salud electrónica para directivos de servicios y sistemas de salud. 9na. ed. Santiago de Chile: Sociedad Española de Informática de la Salud (SEIS); 2012. 

paciente en el servicio de Odontología del Hospital Hermilio Valdizan

5. Toscano J. Investigación del Manejo de las Historias Clínicas Manuales y la aplicación de las Historias Clínicas Electrónicas en Hospitales Públicos de la Ciudad Autónoma de Buenos Aires. Buenos Aires, Argentina. [Maestría]. Universidad de Buenos Aires; 2017.

6. Donato B. La Historia Clínica Electrónica centrada en el paciente como componente fundamental para la gestión de un Sistema de Información de Salud. [Maestría]. Universidad de San Andrés-Argentina; 2013.

7. Cóndor D. Wawared: evaluación de la implementación de una historia clínica electrónica para el control de crecimiento y desarrollo (CRED) del niño. [Maestría]. Universidad Peruana Cayetano Heredia-Perú; 2018.

8. Tuanama J. Adopción de una HCE en establecimientos de salud de primer nivel. [Maestría]. Universidad Peruana Cayetano Heredia-Perú; 2019.

9. Reglamento Ley 30024, Ley del Registro Nacional de Historias Clínicas. Lima: MINSA; 2017.

10. Reglamento de la Ley del Registro Nacional de Historias Clínicas Electrónicas RENHICE. Lima: MINSA; 2015.
11. NTS N¹39-MINSA/2018/DGAIN Norma Técnica de Salud para la Gestión de la historia clínica. Lima: MINSA; 2018.

12. Norma Técnica Nro. 022-MINSA/DGSP-V.01 Norma Técnica de la Historia. Lima: MINSA; 2004.

13. Plan Estratégico Institucional PEI MINSA 2019 2023. Lima: MINSA; 2019.

14. Plan Operativo Institucional 2020. Lima: Hospital Hermilio Valdizán; 2020.

15. Plan Operativo Institucional - POI 2020. Lima: Hospital Hermilio Valdizán; 2020.

16. Veliz L. Propuesta de un sistema informático para mejorar la organización de un Centro Salud SJL, 2016. [Pregrado]. Universidad Wiener-Perú; 2017.

17. Poceiro J. Registro Nacional Historias Clínicas Electrónicas. [Maestría]. Universidad Torcuato de Tella- Argentina; 2014.

18. Uldrich A. Impactos de la salud digital en la atención de la salud. [Internet]. [Citado el 19 de setiembre de 2018]. Disponible en: https://blogs.iadb.org/salud/es/tres-impactosde-la-salud-digital-en-la-atencion-de-la-salud/

Úrsula Gutiérrez Samaniego

ORCID iD: (1) http://orcid.org/0000-0002-6865-7581

ursulamargarita6@gmail.com

Copyright (C) La revista. La revista Kiru es publicada por la Facultad de Odontología de la Universidad de San Martín de Porres, en Lima, Perú. 